\title{
European and North American populations should be screened for coeliac disease
}

\author{
A Fasano
}

Coeliac disease (CD) is an immune mediated enteropathy triggered by ingestion of gluten containing grains in genetically susceptible individuals. ${ }^{1}$ CD is associated with HLA alleles DQ2 and/or DQ8, and in the continued presence of gluten the disease is self perpetuating. ${ }^{12}$ To sustain the position that active screening in search of CD cases is a justified policy, we will review the WHO guidelines for disease mass screening recommendations to establish whether CD satisfies these criteria. ${ }^{3}$

\section{WHO CRITERIA FOR MASS SCREENINGS}

(1) Early detection of the disease could be difficult on a clinical basis.

(2) The disease must be a common disorder causing significant morbidity in the general population.

(3) The screening tests must be highly sensitive and specific for the target disease.

(4) A treatment for the disease must be available.

(5) If not recognised, the disease could result in severe complications difficult to manage.

\section{EARLY DETECTION}

CD can manifest with a previously unsuspected range of clinical presentations, including the typical malabsorption syndrome (chronic diarrhoea, weight loss, abdominal distension) and a spectrum of symptoms potentially affecting any organ or body system. ${ }^{4-6}$ As CD is often atypical or even clinically silent, the vast majority of cases remain undiagnosed for many years and exposed to the risk of long term complications. ${ }^{7}$

\section{PREVALENCE}

Analysis of the world codistribution of the two key components (HLA genes and grains) involved in $C D$ pathogenesis ${ }^{2}$ would predict a wide distribution of the disease, both in regions where $\mathrm{CD}$ is described as a frequent disorder (that is, Europe $)^{6}$ and in areas where the disease has been historically considered rare (that is, the USA). ${ }^{2}$ Recent epidemiological studies conducted in these supposedly "CD free" areas suggest that the disease was indeed under diagnosed ${ }^{89}$ providing evidence that $\mathrm{CD}$ is one of the most frequent genetically based diseases of humankind. Both typical and atypical symptoms are extremely debilitating and, if not diagnosed on time, may lead to irreversible complications, including stunted growth, osteoporosis, autoimmune diseases, or intestinal malignancies. ${ }^{10-14}$

\section{SCREENING TESTS}

With the appreciation that the clinical presentation of CD is more heterogeneous than previously described, more sensitive and specific screening tests have been developed during the past 10-15 years. The discovery of tissue transglutaminase as the autoantigen target of the CD immune response, together with appreciation of the key role of HLA DQ2/DQ8 in CD pathogenesis, led to the development of new diagnostic algorithms that reached sensitivity and specificity close to $100 \% .^{15}$

\section{TREATMENT}

$\mathrm{CD}$ is unique among autoimmune disorders in that a treatment is available. As the pathogenesis of the disease is related to the interplay between genes (both HLA and not HLA) and known environmental factors (that is, gluten containing grains) that trigger the autoimmune process, the cornerstone of CD treatment is a strict gluten free diet. ${ }^{16-18}$ Dietary treatment is associated with rapid symptomatic improvement and healing of the coeliac enteropathy, within a matter of months, and this provides additional diagnostic confirmation.

\section{COMPLICATIONS}

Given the clear role of gluten in causing autoimmunity, CD represents a unique example of an immune mediated disease for which early diagnosis and dietary treatment can prevent its severe, sometimes life threatening, complications. Patients with undiagnosed and untreated CD, as well as those diagnosed later in life, have an increased morbidity and mortality due to associated conditions. ${ }^{11}{ }^{13}$ Since CD is a multisystem disorder, these patients are at risk of chronic ill health, permanent stunted growth, infertility, skeletal disorders, and malignancy. ${ }^{10-14}$ 16-18 $^{1-1}$ These patients also incur increased health care costs because of the multiple subspecialists and multiple laboratory tests performed until the correct diagnosis is obtained. The mortality rate at every age is twofold greater in untreated coeliacs.

\section{FINAL REMARKS}

Does CD fulfil the WHO guidelines for mass screening?

Absolutely, as five out of five major criteria are satisfied.

\section{Why screening for CD?}

Even without a cost-benefit analysis, it is intuitive that an increased awareness of the disease, coupled with a low threshold for serological testing, will uncover a large portion of the submerged CD "iceberg". Due to the high gluten dependent morbidity related to untreated $\mathrm{CD}$ and the unacceptable delay with which CD patients are currently diagnosed, serological screenings are 


\section{Summary}

- Coeliac disease is a common condition in Europe and North America, with a prevalence approaching $1 \%$ in the general population.

- Clinical diagnosis is challenging, given the protean clinical manifestations of the disease. Specific and sensitive serological screening tests are currently available.

- There is a treatment available for the disease.

- If untreated, coeliac disease may result in lifelong complications.

- Currently the "best buy" to screen for the disease is a case finding approach.

justified to prevent both social and personal costs and to increase the quality of life of a multitude of individuals potentially affected by $\mathrm{CD}$.

\section{Who should be screened?}

The need for CD mass screening of the general population will depend on the results of comprehensive, well performed, cost effectiveness analyses. Currently, the best epidemiological approach to look for CD seems to be a systematic process of case finding in which patients with symptoms and/or conditions known to be associated with CD are specifically targeted ${ }^{19}$.

\section{REFERENCES}

1 Sollid LM, McAdam SN, Molberg O, et al. Genes and environment in celiac disease. Acta Odont Scand 2001;59:183-6

2 Fasano A Catassi C. Current approaches to diagnosis and treatment of celiac disease: an evolving spectrum. Gastroenterology 2001;120:636-51.
3 WHO mass screening recommendations: http://www.who.int/en/

4 Ferguson A, Arranz E, O'Mahony S. Clinical and pathological spectrum of coeliac disease-active, silent latent, potential. Gut 1993:34:150-1.

5 Catassi C, Fasano A. New developments in childhood celiac disease. Curr Gastroenterol Rep 2002;4:238-43.

6 Catassi C, Ratsch IM, Fabiani E, et al. Coeliac disease in the year 2000: exploring the iceberg. Lancet 1994;343:200-3.

7 Green P, Stavropoulos S, Panagi S, et al. Characteristics of adult celiac disease in the USA: results of a national survey. Am J Gastroenterol 2001;96:126-31.

8 Not T, Horvath K, Hill ID, et al. Celiac disease risk in the USA: high prevalence of antiendomysium antibodies in healthy blood donors. Scand J Gastroenterol 1998:33:494-8.

9 Hill I, Fasano A, Schwartz R, et al. The prevalence of celiac disease in at-risk groups of children in the United States. J Pediatr 2000;136:86-90.

10 Cellier C, Flobert C, Cormier C, et al. Severe osteopenia in symptom-free adults with a childhood diagnosis of coeliac disease. Lancet 2000;355:806.

11 Corrao G, Corazza GR, Bagnardi V, et al. Mortality in patients with coeliac disease and their relatives: a cohort study. Lancet 2001;358:356-61.

12 Holmes GK, Prior P, Lane MR, et al. Malignancy in coeliac disease-effect of a gluten free diet. Gut 1989:30:333-8.

13 Logan RF, Rifkind EA, Turner ID, et al. Mortality in celiac disease. Gastroenterology 1989;97:265-71.

14 Ventura A, Magazzu G, Greco L. Duration of exposure to gluten and risk for autoimmune disorders in patients with celiac disease. SIGEP Study Group for Autoimmune Disorders in Celiac Disease. Gastroenterology 1999;117:297-303.

15 Fasano A. Celiac disease: the past, the present, the future. Pediatrics 2001;107:768-70.

16 Pietzak MM, Catassi C, Drago S, et al. Celiac disease: going against the grains. Nutr Clin Prac $2001 ; 16: 335-44$.

17 American Gastroenterological Association Medical Position Statement: Celiac sprue. Gastroenterology 2001;120:1522-5.

18 Ciclitira PJ. AGA technical review on celiac sprue. American Gastroenterological Association. Gastroenterology 2001;120:1526-40.

19 Hin H, Bird G, Fisher P, et al. Coeliac disease in primary care: case finding study. BMV 1999;318:164-7. 